\title{
Performance trends in individual medley events during FINA World Master Championships from 1986 to 2014
}

\author{
Nikolaidis, Pantelis T ; Knechtle, Beat
}

\begin{abstract}
BACKGROUND: Performance trends during the last decades in elite individual medley swimmers are known, but not for age groups swimmers. Therefore, the aim of the present study was to examine participation and performance trends in age groups swimmers competing in FINA World Masters Championships (1986-2014) in individual 200m and 400m medley. METHODS: We included all swimmers (women, $\mathrm{n}=6,424 ;$ men, $\mathrm{n}=7,588$ ) for each distance and age groups (25-29 to 95-99 years) in the analysis to avoid a selection bias by limiting to a fixed sample (e.g. the ten fastest). Changes in participation and changes in sex difference across editions were analyzed using linear regression models. Changes in performance across editions were analyzed using a mixed-effects regression model with finisher as random variable to consider finishers who completed several races. Sex, distance and calendar year were included as fixed variables. We also considered interaction effects between sex and distance. The men-to-women ratio was compared using a paired t-test. RESULTS: In 200m, the participation of women increased from 35-39 to 75-79 years and of men from 35-39 to 85-89 years $(p<0.05)$. In $400 \mathrm{~m}$, the participation of women increased from 50-54 to 75-79 years and of men from 60-64 to 85-89 years $(\mathrm{p}<0.05)$. From 25-29 to 90-94 years, women and men improved performance in both editions $(\mathrm{p}<0.05)$. Men were faster than women from 25-29 to 80-84 years $(\mathrm{p}<0.05)$, but not in 85-90 and 90-94 years. Sex and distance showed a significant interaction from $25-29$ to $85-89$ years $(\mathrm{p}<0.05)$, but not in $90-94$ years. Women reduced the gap to men in 40-44 and 45-49 years in 200m, and 40-44 years in $400 \mathrm{~m}(\mathrm{p}<0.05)$. CONCLUSIONS: In summary, the participation increased in older age groups ( $>35$ years), women and men from 25-29 to 90-94 years improved, and men were faster than women from $25-29$ to $80-84$ years, but not from 85-89 to $90-94$ years. These data suggest that master swimmers will continue to increase participation and improve performance in individual medley. The findings of the present study might help coaches and fitness trainers of medley swimmers to tailor their training programs considering the age of their swimmers.
\end{abstract}

DOI: https://doi.org/10.23736/S0022-4707.17.06992-4

Posted at the Zurich Open Repository and Archive, University of Zurich

ZORA URL: https://doi.org/10.5167/uzh-138559

Journal Article

Accepted Version

Originally published at:

Nikolaidis, Pantelis T; Knechtle, Beat (2018). Performance trends in individual medley events during FINA World Master Championships from 1986 to 2014. Journal of Sports Medicine and Physical Fitness, 58(5):690-698.

DOI: https://doi.org/10.23736/S0022-4707.17.06992-4 


\title{
Performance trends in individual medley events during FINA World Master Championships from 1986 to 2014
}

\author{
Pantelis T. NIKOLAIDIS ${ }^{*}$, Beat KNECHTLE 2,3
}

${ }^{1}$ Exercise Physiology Laboratory, Nikaia, Greece; ${ }^{2}$ St. Gallen Health Center, St. Gallen, Switzerland; ${ }^{3}$ Institute of Primary Care, University of Zurich, Zurich, Switzerland

*Corresponding author: Pantelis Nikolaidis, Exercise Physiology Laboratory, Thermopylon 7, Nikaia 18450, Greece. E-mail: pademil@hotmail.com

\section{A B S T R A C T}

BACKGROUND: Performance trends during the last decades in elite individual medley swimmers are known, but not for age groups swimmers. Therefore, the aim of the present study was to examine participation and performance trends in age groups swimmers competing in FINA World Masters Championships (1986-2014) in individual 200-m and 400-m medley.

METHODS: We included all swimmers (6424 women, $7588 \mathrm{men}$ ) for each distance and age groups (25-29 to 95-99 years) in the analysis to avoid a selection bias by limiting to a fixed sample (e.g. the ten fastest). Changes in participation and changes in sex difference across editions were analyzed using linear regression models. Changes in performance across editions were analyzed using a mixed-effects regression model with finisher as random variable to consider finishers who completed several races. Sex, distance and calendar year were included as fixed variables. We also considered interaction effects between sex and distance. The men-to-women ratio was compared using a paired $t$-test.

RESULTS: In $200 \mathrm{~m}$, the participation of women increased from 35-39 to 75-79 years and of men from 35-39 to 85-89 years (P<0.05). In 400 m, the participation of women increased from 50-54 to 75-79 years and of men from 60-64 to 85-89 years $(\mathrm{P}<0.05)$. From 25-29 to 90-94 years, women and men improved performance in both editions $(\mathrm{P}<0.05)$. Men were faster than women from 25-29 to 80-84 years $(\mathrm{P}<0.05)$, but not in 85-90 and 90-94 years. Sex and distance showed a significant interaction from 25-29 to 85-89 years $(\mathrm{P}<0.05)$, but not in 90-94 years. Women reduced the gap to men in 40-44 and 45-49 years in $200 \mathrm{~m}$, and $40-44$ years in $400 \mathrm{~m}(\mathrm{P}<0.05)$.

CONCLUSIONS: In summary, the participation increased in older age groups ( $>35$ years), women and men from 25-29 to 90-94 years improved, and men were faster than women from 25-29 to 80-84 years, but not from 85-89 to 90-94 years. These data suggest that master swimmers will continue to increase participation and improve performance in individual medley. The findings of the present study might help coaches and fitness trainers of medley swimmers to tailor their training programs considering the age of their swimmers.

(Cite this article as: Nikolaidis PT, Knechtle B. Performance trends in individual medley events during FINA World Master Championships from 1986 to 2014.

Key words: Athletes - Swimming - Athletic performance - Sex characteristics - Aging.

Swimming can be performed in the four major strokes such as freestyle, backstroke, breaststroke and butterfly. The combination of the four strokes in one single race is called individual medley. ${ }^{1}$ Each stroke has an equal part of the overall distance, i.e. a quarter of the overall distance is swum in each stroke. The strokes are swum in the order butterfly, backstroke, breaststroke and freestyle. ${ }^{1}$ Usually, individual medley is held over $200 \mathrm{~m}$ and $400 \mathrm{~m}$. The speed in individual medley is determined by kinematic parameters and technical skills. ${ }^{2}$ The performance in the individual medley in both 200-m and 400-m events is influenced by the course length of the swimming pool with faster times being observed in the $25-\mathrm{m}$ than in the $50-\mathrm{m}$ course length. ${ }^{3}$

Improvements in swimming performance in the four major strokes have been well documented for elite swimmers competing at world class level (e.g. World Championships, Olympic Games).4-6 For elite individual medley swimmers, the changes in performance 
across years, ${ }^{7}$ the age of the best performance $4,8,9$ and the pacing strategy 10 have already been investigated.

The FINA holds apart from World Championships for elite swimmers since 1986 biannually the World Masters Championships for all disciplines and distances in pool and open-water swimming. ${ }^{1}$ In master swimmers, it is known that swimming performance decreases with increasing age, 11,12 but few data exist for changes in performance across years. ${ }^{12}$ Mainly freestyle swimming has been investigated in age group swimmers. However, little is known for age group freestyle swimmers for distances from $100 \mathrm{~m}$ to $800 \mathrm{~m}$ or longer. ${ }^{13}$ Akkari et al. ${ }^{13}$ recently investigated changes in swimming performance in master swimmers competing in 100-m freestyle between 1975 and 2013.

However, trends in participation, performance and sex difference in performance of age group individual medley swimmers, are not known. Such knowledge would help coaches and trainers working with swimmers who compete in medley developing sex- and age-tailored training programs. Therefore, the aim of the present study was to investigate trends in changes of participation and performance in master swimmers competing in the FINA World Masters Championships held between 1986 and 2014 in $200 \mathrm{~m}$ and 400m individual medley. We hypothesized that participation would increase and performance would improve in these age group swimmers as it has been shown for age group competing in other sports disciplines such as marathon ${ }^{14-16}$ and ultramarathon running. ${ }^{17}$

\section{Materials and method}

This study was approved by the Institutional Review Board of St. Gallen, Switzerland, with waiver of the requirement for informed consent given that the study involved the analysis of publicly available data.

All data were obtained from the official and free accessible FINA website. ${ }^{1}$ FINA defines master swimmers as those equal or older than 25 years and records all competitors in 5-years age groups from 25-29 years to $105-109$ years. All swimmers (6424 women, 7588 men) competing in all age groups in the FINA World Masters Championships in 200-m and 400-m individual medley between 1986 and 2014 (Table I) were analyzed for trends in participation, performance and sex difference in performance. Figure 1 summarizes the number
TABLE I._FINA World Master Championships from 1986 to 2014.

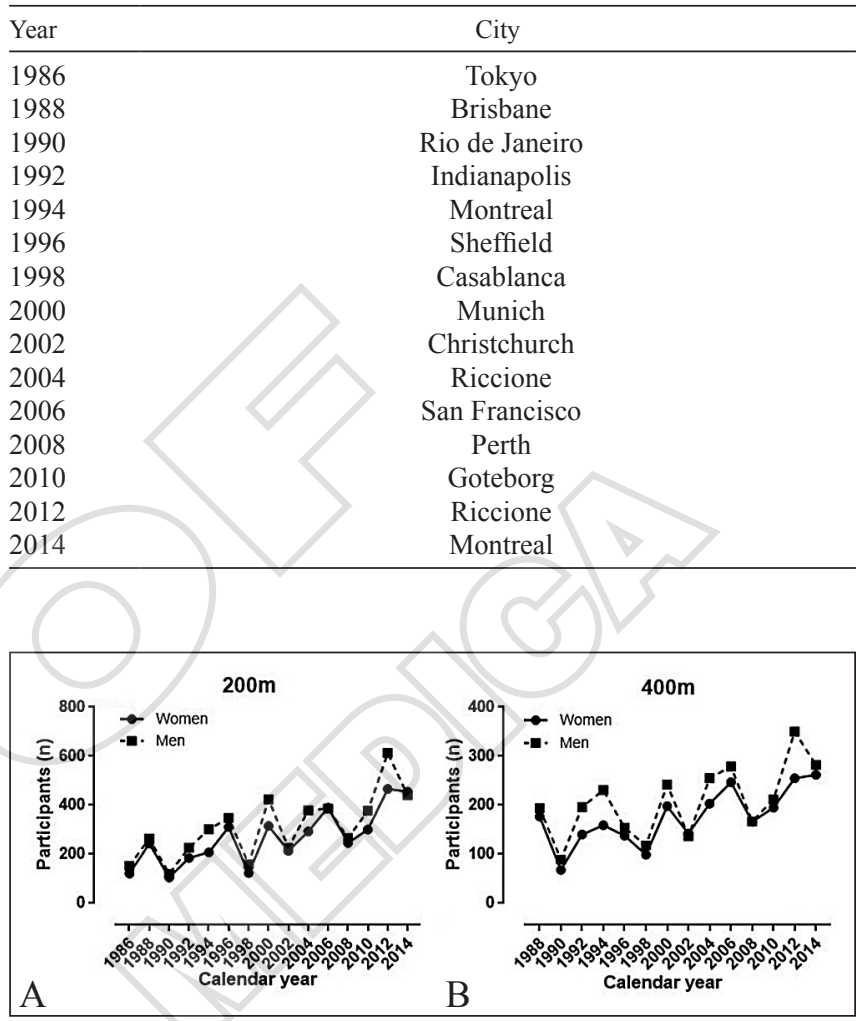

Figure 1.-Overall and by sex participation for 200-m (A) and 400-m medley (B) in FINA World Master Championships by calendar year.

of overall, women and men finishers for both 200-m and 400-m individual medley for each edition. For all ranked competitors, mean race time was calculated for all age groups for each year. The men-to-women ratio was calculated by dividing the number of men competitors by the number of women competitors for age groups and editions. Sex difference was calculated using the formula $100 \times[$ (race time in women $)-($ race time in men $) /$ (race time in men)].

\section{Statistical analysis}

To avoid a selection bias by limiting to a fixed sample (e.g. the ten fastest), we included all swimmers for each distance and age groups in the analysis. Changes in participation and changes in sex difference across editions were analyzed using linear regression models. Changes in performance across editions were analyzed using a mixed-effects regression model with participant identi- 
fication (i.e. name of the swimmer) as random variable to consider swimmers who completed several races in several editions. Sex, distance and calendar year were included as fixed variables and race time was the dependent variable. We also considered interaction effects between sex and distance. A two-way analysis of variance examined the effect of sex and calendar year on race time for each race distance. The magnitude of these effects was examined using eta squared $\left(\eta^{2}\right)$ evaluated as following: small $\left(0.010<\eta^{2} \leq 0.059\right)$, moderate $\left(0.059<\eta^{2} \leq 0.138\right)$ and large $\left(\eta^{2}>0.138\right) .{ }^{18}$ The mento-women ratio was compared using a paired $t$-test. Statistical analyses were performed using IBM SPSS Statistics (v. 22, IBM SPSS, Chicago, IL, USA) and GraphPad Prism (v. 6.01, GraphPad Software, La Jolla, CA, USA). Significance was accepted at $\mathrm{P}<0.05$ (twosided for $t$-tests). Data in the text and tables are given as mean \pm standard deviation.

\section{Results}

\section{Participation trends}

Between 1986 and 2014, a total of 14,012 swimmers were analyzed. In 200-m individual medley, 3975 women and 4682 men were classified between 1986 and 2014. In 400-m individual medley, 2449 women and 2906 men were recorded from 1988 to 2014. Figure 2 presents the trend in participation for women and men for $200 \mathrm{~m}$ and $400 \mathrm{~m}$. In $200 \mathrm{~m}$, participation increased

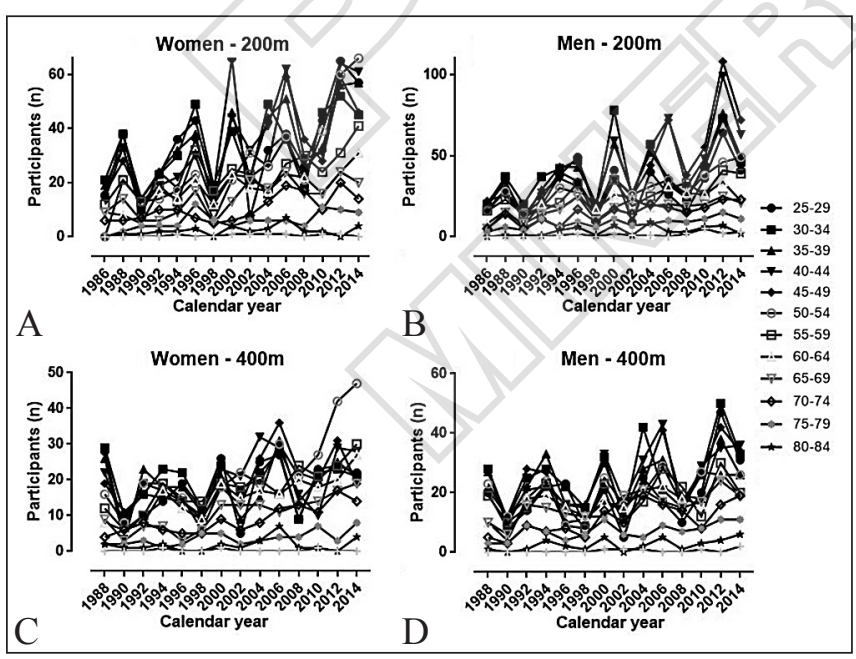

Figure 2.-A-D) Participation of women and men by age group for $200 \mathrm{~m}$ and $400 \mathrm{~m}$.

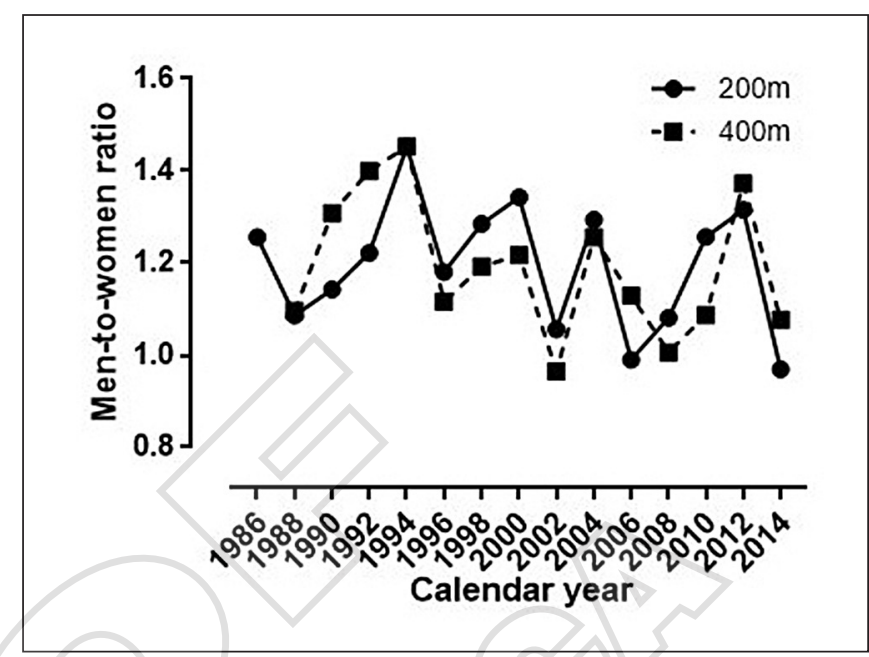

Figure 3.-The men-to-women ratio across editions for $200 \mathrm{~m}$ and $400 \mathrm{~m}$.

in women in age groups 35-39 to 75-79 years and in men in age groups 35-39 to 85-89 years. In $400 \mathrm{~m}$, participation increased in women in age groups 50-54 to 75-79 years. In men, participation increased in age groups 60 64 to 85-89 years. Figure 3 shows the men-to-women ratio for 200-m and 400-m individual medley across editions. The men-to-women ratio remained unchanged in $200 \mathrm{~m}\left(r^{2}=0.05, \mathrm{P}=0.44\right)$ and $400 \mathrm{~m}\left(r^{2}=0.04, \mathrm{P}=0.49\right)$. In $200 \mathrm{~m}$ and $400 \mathrm{~m}$, more men than women competed $(\mathrm{P}<0.05)$. Figure 4 presented the men-to-women ratio by age groups.

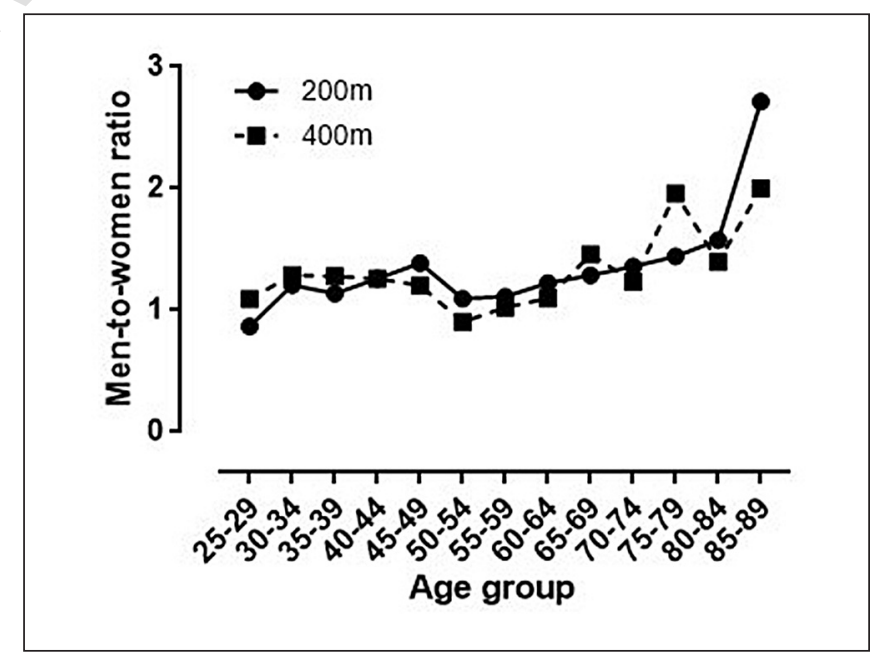

Figure 4.- Men-to-women ratio by age group for $200 \mathrm{~m}$ and $400 \mathrm{~m}$. 
TABLE II.-_Race times (min:sec) for women in age groups 25-29 to 90-94 years for $200 \mathrm{~m}$ and $400 \mathrm{~m}$ between 1986 and 2014.

\begin{tabular}{|c|c|c|c|c|c|c|c|c|}
\hline Distance & Age group & 1986 & 1988 & 1990 & 1992 & 1994 & 1996 & 1998 \\
\hline \multirow[t]{14}{*}{$200 \mathrm{~m}$} & $25-29$ & $3: 08 \pm 0: 26$ & $2: 56 \pm 0: 16$ & $3: 03 \pm 0: 18$ & $2: 50 \pm 0: 14$ & $3: 00 \pm 0: 20$ & $2: 55 \pm 0: 17$ & $2: 48 \pm 0: 08$ \\
\hline & $30-34$ & $3: 20 \pm 0: 27$ & $3: 04 \pm 0: 24$ & $3: 09 \pm 0: 23$ & $2: 51 \pm 0: 15$ & $3: 08 \pm 0: 21$ & $2: 56 \pm 0: 18$ & $2: 47 \pm 0: 09$ \\
\hline & $35-39$ & $3: 38 \pm 0: 26$ & $3: 26 \pm 0: 25$ & $3: 05 \pm 0: 20$ & $2: 58 \pm 0: 42$ & $3: 08 \pm 0: 23$ & $3: 04 \pm 0: 18$ & $2: 53 \pm 0: 12$ \\
\hline & $40-44$ & $3: 42 \pm 0: 23$ & $3: 31 \pm 0: 26$ & $3: 17 \pm 0: 22$ & $3: 26 \pm 0: 42$ & $3: 44 \pm 0: 32$ & $3: 22 \pm 0: 26$ & $3: 03 \pm 0: 21$ \\
\hline & $45-49$ & $3: 52 \pm 0: 29$ & $3: 41 \pm 0: 27$ & $3: 35 \pm 0: 31$ & $3: 27 \pm 0: 27$ & $3: 33 \pm 0: 29$ & $3: 26 \pm 0: 27$ & $3: 11 \pm 0: 18$ \\
\hline & $50-54$ & & $4: 12 \pm 0: 36$ & $3: 57 \pm 0: 39$ & $3: 39 \pm 0: 24$ & $3: 51 \pm 0: 30$ & $3: 38 \pm 0: 32$ & $3: 19 \pm 0: 21$ \\
\hline & $55-59$ & $4: 02 \pm 0: 23$ & $4: 22 \pm 0: 46$ & $4: 37 \pm 0: 41$ & $4: 03 \pm 0: 30$ & $3: 58 \pm 0: 28$ & $3: 54 \pm 0: 28$ & $3: 38 \pm 0: 18$ \\
\hline & $60-64$ & $4: 16 \pm 0: 36$ & $4: 15 \pm 0: 29$ & $3: 56 \pm 0: 25$ & $4: 36 \pm 1: 02$ & $4: 16 \pm 0: 49$ & $4: 17 \pm 0: 39$ & $3: 57 \pm 0: 17$ \\
\hline & $65-69$ & $5: 14 \pm 0: 60$ & $4: 32 \pm 0: 21$ & $4: 59 \pm 1: 21$ & $4: 07 \pm 0: 29$ & $4: 19 \pm 0: 40$ & $4: 43 \pm 0: 38$ & $4: 31 \pm 0: 32$ \\
\hline & $70-74$ & $5: 33 \pm 1: 19$ & $5: 19 \pm 1: 02$ & $5: 41 \pm 1: 23$ & $5: 27 \pm 1: 47$ & $5: 57 \pm 1: 06$ & $4: 33 \pm 0: 42$ & $4: 45 \pm 0: 48$ \\
\hline & $75-79$ & & $7: 24 \pm 2: 25$ & $5: 21 \pm 0: 49$ & $5: 33 \pm 0: 57$ & $6: 19 \pm 2: 26$ & $5: 50 \pm 1: 11$ & $5: 51 \pm 1: 00$ \\
\hline & $80-84$ & & $6: 55$ & $7: 26$ & $6: 38 \pm 1: 26$ & $5: 05 \pm 0: 11$ & $5: 47 \pm 0: 58$ & \\
\hline & $85-89$ & & & & & 10:59 & & \\
\hline & $90-94$ & & & & & & & \\
\hline \multirow[t]{14}{*}{$400 \mathrm{~m}$} & $25-29$ & & $6: 02 \pm 0: 32$ & $5: 50 \pm 0: 11$ & $5: 52 \pm 0: 1$ & $6: 04 \pm 0: 30$ & $6: 01 \pm 0: 31$ & $5: 55 \pm 0: 21$ \\
\hline & $30-34$ & & $6: 38 \pm 0: 49$ & $6: 32 \pm 0: 47$ & $0: 56$ & $6: 09 \pm 0: 48$ & $6: 03 \pm 0: 29$ & $5: 57 \pm 0: 20$ \\
\hline & $35-39$ & & $6: 51 \pm 0: 42$ & $7: 08 \pm 0: 50$ & $6: 30 \pm 0: 41$ & $6: 27 \pm 0: 42$ & $6: 28 \pm 0: 38$ & $6: 13 \pm 0: 24$ \\
\hline & $40-44$ & & $7: 37 \pm 0: 54$ & $7: 13 \pm 0: 36$ & $7: 10 \pm 0: 52$ & $7: 29 \pm 1: 01$ & $6: 55 \pm 0: 32$ & $6: 37 \pm 0: 38$ \\
\hline & $45-49$ & & $7: 33 \pm 0: 44$ & $7: 38 \pm 0: 59$ & $7: 07 \pm 0: 58$ & $7: 38 \pm 1: 04$ & $7: 00 \pm 0: 32$ & $6: 49 \pm 0: 48$ \\
\hline & $50-54$ & & $8: 38 \pm 1: 21$ & $8: 49 \pm 1: 21$ & $7: 51 \pm 0: 52$ & $8: 13 \pm 0: 58$ & $7: 28 \pm 0: 30$ & $7: 03 \pm 0: 34$ \\
\hline & $55-59$ & & $8: 42 \pm 1: 02$ & $9: 48 \pm 1: 14$ & $8: 03 \pm 0: 46$ & $8: 33 \pm 1: 12$ & $7: 57 \pm 0: 39$ & $7: 41 \pm 0: 44$ \\
\hline & $60-64$ & & $9: 37 \pm 1: 25$ & $8: 39 \pm 1: 09$ & $9: 36 \pm 1: 33$ & $8: 55 \pm 1: 14$ & $8: 24 \pm 0: 44$ & $8: 22 \pm 0: 53$ \\
\hline & $65-69$ & & $9: 52 \pm 1: 17$ & $9: 13 \pm 1: 05$ & $9: 07 \pm 1: 13$ & $9: 13 \pm 1: 04$ & $9: 26 \pm 0: 35$ & $9: 42 \pm 0: 37$ \\
\hline & $70-74$ & & $12: 48 \pm 2: 32$ & $11: 58 \pm 3: 06$ & $11: 47 \pm 3: 05$ & $11: 31 \pm 2: 43$ & $8: 47 \pm 1: 02$ & $10: 20 \pm 1: 57$ \\
\hline & $75-79$ & & $15: 36 \pm 4: 27$ & $12: 45 \pm 0: 20$ & $12: 12 \pm 1: 35$ & $20: 29$ & $10: 42 \pm 1: 20$ & $12: 44 \pm 2: 54$ \\
\hline & $80-84$ & & $13: 45 \pm 0: 04$ & $16: 08$ & $11: 57$ & $00 \pm 0: 05$ & & \\
\hline & $85-89$ & & & & & & & \\
\hline & $90-94$ & & & & & & & \\
\hline
\end{tabular}

TABLE III.-Race times (min:sec) for men in age groups 25-29 to 90-94 years for $200 \mathrm{~m}$ and $400 \mathrm{~m}$ between 1986 and 2014.

\begin{tabular}{|c|c|c|c|c|c|c|c|c|}
\hline Distance & Age group & 1986 & 1988 & 1990 & 1992 & 1994 & 1996 & 1998 \\
\hline \multirow[t]{14}{*}{$200 \mathrm{~m}$} & $25-29$ & $2: 37 \pm 0: 11$ & $2: 40 \pm 0: 26$ & $2: 32 \pm 0: 05$ & $2: 30 \pm 0: 11$ & $2: 39 \pm 0: 18$ & $2: 31 \pm 0: 12$ & $2: 33 \pm 0: 08$ \\
\hline & $30-34$ & $2: 49 \pm 0: 21$ & $2: 42 \pm 0: 23$ & $2: 37 \pm 0: 15$ & $2: 30 \pm 0: 10$ & $2: 44 \pm 0: 21$ & $2: 35 \pm 0: 10$ & $2: 34 \pm 0: 11$ \\
\hline & $35-39$ & $2: 54 \pm 0: 20$ & $2: 54 \pm 0: 22$ & $2: 44 \pm 0: 13$ & $2: 40 \pm 0: 19$ & $2: 50 \pm 0: 18$ & $2: 45 \pm 0: 20$ & $2: 28 \pm 0: 08$ \\
\hline & $40-44$ & & $2: 57 \pm 0: 22$ & $2: 50 \pm 0: 23$ & $2: 46 \pm 0: 17$ & $2: 49 \pm 0: 22$ & $2: 47 \pm 0: 16$ & $2: 42 \pm 0: 12$ \\
\hline & $45-49$ & & $3: 02 \pm 0: 24$ & $2: 52 \pm 0: 14$ & $2: 55 \pm 0: 28$ & $3: 06 \pm 0: 26$ & $2: 56 \pm 0: 21$ & $2: 47 \pm 0: 13$ \\
\hline & $50-54$ & $\pm 0: 22$ & $3: 22 \pm 0: 31$ & $3: 14 \pm 0: 22$ & $3: 01 \pm 0: 20$ & $3: 11 \pm 0: 27$ & $3: 05 \pm 0: 25$ & $2: 51 \pm 0: 11$ \\
\hline & $55-59$ & $3: 42 \pm 0: 45$ & $3: 27 \pm 0: 26$ & $3: 34 \pm 0: 35$ & $3: 16 \pm 0: 21$ & $3: 14 \pm 0: 19$ & $3: 22 \pm 0: 31$ & $3: 00 \pm 0: 17$ \\
\hline & $60-64$ & $3: 49 \pm 0: 32$ & $3: 31 \pm 0: 21$ & $3: 29 \pm 0: 16$ & $3: 24 \pm 0: 18$ & $3: 32 \pm 0: 38$ & $3: 26 \pm 0: 29$ & $3: 17 \pm 0: 16$ \\
\hline & $65-69$ & $4: 20 \pm 0: 55$ & $3: 43 \pm 0: 19$ & $4: 35 \pm 0: 41$ & $3: 40 \pm 0: 24$ & $3: 54 \pm 0: 44$ & $3: 33 \pm 0: 22$ & $3: 38 \pm 0: 20$ \\
\hline & $70-74$ & $4: 25 \pm 0: 25$ & $4: 08 \pm 0: 35$ & $4: 19 \pm 0: 36$ & $4: 26 \pm 0: 44$ & $4: 18 \pm 0: 20$ & $3: 53 \pm 0: 30$ & $3: 41 \pm 0: 19$ \\
\hline & $75-79$ & $6: 03 \pm 1: 37$ & & $4: 25 \pm 0: 19$ & $4: 25 \pm 0: 38$ & $4: 30 \pm 0: 44$ & $4: 18 \pm 0: 36$ & $4: 41 \pm 0: 55$ \\
\hline & $80-84$ & & & $6: 41$ & $5: 03$ & $5: 05 \pm 1: 06$ & $5: 15 \pm 1: 16$ & $4: 47$ \\
\hline & $85-89$ & & & & & $5: 07$ & $5: 22 \pm 0: 07$ & \\
\hline & $90-94$ & & & & & & $7: 08$ & \\
\hline \multirow[t]{14}{*}{$400 \mathrm{~m}$} & $25-29$ & & $5: 27 \pm 0: 30$ & $5: 38 \pm 0: 23$ & $5: 19 \pm 0: 34$ & $5: 37 \pm 0: 43$ & $5: 18 \pm 0: 25$ & $5: 29 \pm 0: 24$ \\
\hline & $30-34$ & & $5: 45 \pm 0: 45$ & $5: 31 \pm 0: 24$ & $5: 20 \pm 0: 20$ & $5: 33 \pm 0: 31$ & $5: 33 \pm 0: 25$ & $5: 29 \pm 0: 27$ \\
\hline & $35-39$ & & $6: 20 \pm 0: 50$ & $6: 03 \pm 0: 42$ & $5: 37 \pm 0: 33$ & $5: 56 \pm 0: 44$ & $5: 44 \pm 0: 26$ & $5: 29 \pm 0: 25$ \\
\hline & $40-44$ & & $6: 24 \pm 0: 46$ & $5: 54 \pm 0: 41$ & $5: 56 \pm 0: 44$ & $6: 03 \pm 0: 51$ & $5: 57 \pm 0: 30$ & $5: 50 \pm 0: 26$ \\
\hline & $45-49$ & & $6: 40 \pm 0: 58$ & $6: 16 \pm 0: 31$ & $6: 34 \pm 1: 02$ & $6: 31 \pm 0: 55$ & $6: 19 \pm 0: 28$ & $6: 03 \pm 0: 29$ \\
\hline & $50-54$ & & $7: 17 \pm 1: 31$ & $6: 43 \pm 0: 31$ & $6: 40 \pm 0: 44$ & $6: 56 \pm 1: 07$ & $6: 09 \pm 0: 26$ & $6: 10 \pm 0: 33$ \\
\hline & $55-59$ & & $7: 26 \pm 0: 48$ & $7: 07 \pm 0: 50$ & $7: 04 \pm 0: 39$ & $7: 17 \pm 0: 45$ & $6: 42 \pm 0: 35$ & $6: 31 \pm 0: 48$ \\
\hline & $60-64$ & & $7: 42 \pm 1: 00$ & $7: 31 \pm 0: 35$ & $7: 27 \pm 0: 41$ & $7: 45 \pm 0: 58$ & $7: 21 \pm 0: 36$ & $7: 02 \pm 0: 40$ \\
\hline & $65-69$ & & $8: 42 \pm 1: 48$ & $8: 53 \pm 1: 17$ & $7: 58 \pm 0: 54$ & $8: 13 \pm 1: 02$ & $7: 36 \pm 0: 51$ & $7: 56 \pm 0: 36$ \\
\hline & $70-74$ & & $8: 30 \pm 0: 57$ & $9: 15 \pm 1: 48$ & $8: 53 \pm 1: 48$ & $9: 38 \pm 2: 00$ & $8: 33 \pm 0: 52$ & $8: 18 \pm 1: 00$ \\
\hline & $75-79$ & & $11: 09 \pm 3: 04$ & $10: 29 \pm 1: 40$ & $9: 18 \pm 1: 24$ & $9: 18 \pm 1: 52$ & $8: 37 \pm 0: 49$ & $10: 57 \pm 2: 30$ \\
\hline & $80-84$ & & $9: 47$ & & $10: 58$ & $13: 23 \pm 2: 29$ & $9: 15 \pm 1: 23$ & $10: 33$ \\
\hline & $85-89$ & & & & & & & \\
\hline & $90-94$ & & & & & & & \\
\hline
\end{tabular}




\begin{tabular}{|c|c|c|c|c|c|c|c|}
\hline 2000 & 2002 & 2004 & 2006 & 2008 & 2010 & 2012 & 2014 \\
\hline $2: 52 \pm 0: 10$ & $2: 56 \pm 0: 14$ & $2: 46 \pm 0: 06$ & $2: 46 \pm 0: 09$ & $2: 46 \pm 0: 11$ & $2: 49 \pm 0: 09$ & $2: 46 \pm 0: 08$ & $2: 47 \pm 0: 09$ \\
\hline $2: 53 \pm 0: 11$ & $2: 52 \pm 0: 14$ & $2: 48 \pm 0: 12$ & $2: 49 \pm 0: 10$ & $2: 51 \pm 0: 11$ & $2: 48 \pm 0: 11$ & $2: 47 \pm 0: 09$ & $2: 49 \pm 0: 11$ \\
\hline $3: 04 \pm 0: 18$ & $3: 00 \pm 0: 21$ & $2: 56 \pm 0: 13$ & $2: 54 \pm 0: 12$ & $2: 58 \pm 0: 12$ & $2: 52 \pm 0: 13$ & $2: 53 \pm 0: 12$ & $2: 54 \pm 0: 13$ \\
\hline $3: 11 \pm 0: 19$ & $3: 03 \pm 0: 20$ & $3: 01 \pm 0: 12$ & $2: 56 \pm 0: 14$ & $2: 59 \pm 0: 18$ & $3: 04 \pm 0: 14$ & $2: 56 \pm 0: 13$ & $2: 57 \pm 0: 15$ \\
\hline $3: 17 \pm 0: 24$ & $3: 18 \pm 0: 16$ & $3: 04 \pm 0: 14$ & $3: 06 \pm 0: 16$ & $3: 09 \pm 0: 20$ & $3: 02 \pm 0: 18$ & $3: 01 \pm 0: 13$ & $3: 06 \pm 0: 16$ \\
\hline $3: 37 \pm 0: 29$ & $3: 35 \pm 0: 24$ & $3: 20 \pm 0: 18$ & $3: 19 \pm 0: 18$ & $3: 14 \pm 0: 19$ & $3: 15 \pm 0: 17$ & $3: 16 \pm 0: 17$ & $3: 15 \pm 0: 19$ \\
\hline $3: 48 \pm 0: 26$ & $3: 40 \pm 0: 23$ & $3: 37 \pm 0: 19$ & $3: 29 \pm 0: 18$ & $3: 34 \pm 0: 19$ & $3: 31 \pm 0: 18$ & $3: 24 \pm 0: 20$ & $3: 23 \pm 0: 20$ \\
\hline $4: 02 \pm 0: 38$ & $3: 50 \pm 0: 28$ & $3: 41 \pm 0: 20$ & $3: 41 \pm 0: 25$ & $3: 45 \pm 0: 26$ & $3: 31 \pm 0: 23$ & $3: 40 \pm 0: 21$ & $3: 34 \pm 0: 23$ \\
\hline $4: 30 \pm 0: 43$ & $4: 23 \pm 0: 24$ & $4: 12 \pm 0: 20$ & $4: 01 \pm 0: 23$ & $4: 11 \pm 0: 59$ & $4: 03 \pm 0: 27$ & $3: 59 \pm 0: 27$ & $3: 43 \pm 0: 22$ \\
\hline $4: 40 \pm 0: 47$ & $4: 28 \pm 0: 47$ & $4: 32 \pm 0: 23$ & $4: 23 \pm 0: 20$ & $4: 25 \pm 0: 30$ & $4: 14 \pm 0: 28$ & $4: 16 \pm 0: 23$ & $4: 15 \pm 0: 19$ \\
\hline $5: 42 \pm 1: 00$ & $5: 02 \pm 0: 44$ & $4: 57 \pm 0: 44$ & $4: 24 \pm 0: 32$ & $5: 09 \pm 0: 50$ & $5: 02 \pm 0: 34$ & $5: 01 \pm 0: 36$ & $4: 44 \pm 0: 32$ \\
\hline $6: 05 \pm 0: 55$ & $5: 35 \pm 0: 17$ & $5: 43 \pm 0: 55$ & $5: 45 \pm 0: 42$ & $6: 38 \pm 0: 10$ & $4: 28 \pm 0: 03$ & & $5: 04 \pm 0: 47$ \\
\hline $5: 39$ & $6: 18$ & $6: 22$ & $5: 51$ & & 6:02 & $5: 22$ & \\
\hline $5: 59 \pm 0: 22$ & $5: 44 \pm 0: 09$ & $5: 53 \pm 0: 16$ & $5: 52 \pm 0: 19$ & $5: 44 \pm 0: 13$ & $1 \pm 0: 18$ & $5: 45 \pm 0: 17$ & $\begin{array}{l}7: 16 \pm 0: 42 \\
5: 57 \pm 0: 23\end{array}$ \\
\hline $5: 52 \pm 0: 23$ & $5: 51 \pm 0: 27$ & $5: 49 \pm 0: 23$ & $6: 03 \pm 0: 22$ & $5: 55 \pm 0: 23$ & $5: 50 \pm 0: 24$ & $5: 56 \pm 0: 17$ & $6: 01 \pm 0: 26$ \\
\hline $6: 05 \pm 0: 27$ & $6: 05 \pm 0: 40$ & $6: 09 \pm 0: 26$ & $6: 02 \pm 0: 25$ & $6: 15 \pm 0: 27$ & $5: 52 \pm 0: 22$ & $5: 57 \pm 0: 22$ & $6: 05 \pm 0: 33$ \\
\hline $6: 46 \pm 0: 43$ & $6: 20 \pm 0: 34$ & $6: 21 \pm 0: 26$ & $6: 05 \pm 0: 32$ & $6: 04 \pm 0: 31$ & $6: 24 \pm 0: 32$ & $6: 08 \pm 0: 26$ & $6: 05 \pm 0: 30$ \\
\hline $6: 43 \pm 0: 33$ & $6: 59 \pm 0: 38$ & $6: 39 \pm 0: 34$ & $6: 28 \pm 0: 31$ & $6: 38 \pm 0: 39$ & $6: 38 \pm 0: 42$ & $6: 26 \pm 0: 27$ & $6: 23 \pm 0: 29$ \\
\hline $7: 28 \pm 0: 57$ & $7: 24 \pm 0: 46$ & $7: 06 \pm 0: 47$ & $6: 56 \pm 0: 32$ & $7: 00 \pm 0: 44$ & $6: 53 \pm 0: 41$ & $6: 58 \pm 0: 43$ & $6: 54 \pm 0: 46$ \\
\hline $7: 56 \pm 0: 43$ & $7: 45 \pm 0: 46$ & $7: 41 \pm 0: 46$ & $7: 28 \pm 0: 45$ & $7: 44 \pm 0: 48$ & $7: 31 \pm 0: 50$ & $7: 06 \pm 0: 44$ & $7: 09 \pm 0: 43$ \\
\hline $8: 20 \pm 0: 41$ & $8: 30 \pm 0: 59$ & $8: 06 \pm 0: 42$ & $8: 12 \pm 0: 46$ & $8: 01 \pm 0: 57$ & $7: 40 \pm 0: 56$ & $7: 58 \pm 0: 48$ & $7: 44 \pm 0: 54$ \\
\hline $9: 26 \pm 0: 59$ & $9: 12 \pm 1: 00$ & $8: 54 \pm 0: 45$ & $8: 49 \pm 0: 55$ & $8: 51 \pm 0: 47$ & $8: 56 \pm 0: 59$ & $8: 07 \pm 0: 42$ & $8: 03 \pm 0: 51$ \\
\hline $9: 56 \pm 1: 26$ & $9: 22 \pm 1: 18$ & $9: 24 \pm 0: 56$ & $9: 05 \pm 0: 49$ & $10: 00 \pm 1: 09$ & $10: 03 \pm 1: 26$ & $9: 23 \pm 0: 59$ & $9: 06 \pm 1: 01$ \\
\hline $12: 37 \pm 2: 14$ & $8: 52 \pm 0: 35$ & $11: 32 \pm 2: 57$ & $9: 34 \pm 1: 07$ & $12: 06 \pm 2: 20$ & $11: 25 \pm 2: 20$ & $10: 02 \pm 2: 13$ & $9: 57 \pm 1: 28$ \\
\hline \multicolumn{8}{|l|}{ 20:01 } \\
\hline & & & & & & & $15: 21 \pm 1: 37$ \\
\hline 2000 & 2002 & 004 & 2006 & 2008 & 2010 & 2012 & 2014 \\
\hline $2: 33 \pm 0: 08$ & $2: 31 \pm 0: 09$ & $2: 26 \pm 0: 07$ & $2: 29 \pm 0: 07$ & $2: 29 \pm 0: 09$ & $2: 26 \pm 0: 08$ & $2: 25 \pm 0: 05$ & $2: 25 \pm 0: 08$ \\
\hline $2: 35 \pm 0: 10$ & $2: 36 \pm 0: 12$ & $2: 31 \pm 0: 07$ & $2: 27 \pm 0: 09$ & $2: 29 \pm 0: 08$ & $2: 29 \pm 0: 09$ & $2: 30 \pm 0: 06$ & $2: 29 \pm 0: 09$ \\
\hline $2: 37 \pm 0: 11$ & $2: 38 \pm 0: 13$ & $2: 35 \pm 0: 08$ & $2: 30 \pm 0: 07$ & $2: 34 \pm 0: 13$ & $2: 33 \pm 0: 09$ & $2: 32 \pm 0: 08$ & $2: 33 \pm 0: 08$ \\
\hline $2: 42 \pm 0: 13$ & $2: 44 \pm 0: 15$ & $2: 37 \pm 0: 10$ & $2: 35 \pm 0: 09$ & $2: 37 \pm 0: 14$ & $2: 37 \pm 0: 10$ & $2: 39 \pm 0: 09$ & $2: 37 \pm 0: 10$ \\
\hline $2: 49 \pm 0: 14$ & $2: 49 \pm 0: 13$ & $2: 42 \pm 0: 12$ & $2: 39 \pm 0: 12$ & $2: 48 \pm 0: 16$ & $2: 39 \pm 0: 12$ & $2: 42 \pm 0: 11$ & $2: 41 \pm 0: 12$ \\
\hline $2: 58 \pm 0: 14$ & $3: 00 \pm 0: 21$ & $2: 47 \pm 0: 11$ & & $2: 48 \pm 0: 15$ & $2: 43 \pm 0: 12$ & $2: 46 \pm 0: 12$ & $2: 44 \pm 0: 12$ \\
\hline $3: 04 \pm 0: 16$ & $3: 10 \pm 0: 22$ & $2: 56 \pm 0: 11$ & $2: 54 \pm 0: 13$ & $2: 56 \pm 0: 18$ & $2: 54 \pm 0: 12$ & $2: 53 \pm 0: 12$ & $2: 53 \pm 0: 13$ \\
\hline $3: 17 \pm 0: 18$ & $3: 24 \pm 0: 24$ & $3: 05 \pm 0: 14$ & $3: 04 \pm 0: 17$ & $3: 07 \pm 0: 21$ & $3: 04 \pm 0: 16$ & $3: 05 \pm 0: 21$ & $3: 03 \pm 0: 13$ \\
\hline $3: 34 \pm 0: 25$ & $3: 31 \pm 0: 28$ & $3: 23 \pm 0: 16$ & $3: 23 \pm 0: 20$ & $3: 24 \pm 0: 23$ & $3: 16 \pm 0: 18$ & $3: 15 \pm 0: 17$ & $3: 10 \pm 0: 19$ \\
\hline $3: 57 \pm 0: 22$ & $3: 54 \pm 0: 28$ & $3: 44 \pm 0: 17$ & $3: 29 \pm 0: 15$ & $3: 38 \pm 0: 27$ & $3: 35 \pm 0: 23$ & $3: 35 \pm 0: 19$ & $3: 37 \pm 0: 20$ \\
\hline $4: 25 \pm 0: 53$ & $4: 00 \pm 0: 23$ & $3: 53 \pm 0: 21$ & $3: 53 \pm 0: 20$ & $3: 53 \pm 0: 30$ & $3: 40 \pm 0: 26$ & $3: 44 \pm 0: 25$ & $4: 09 \pm 0: 27$ \\
\hline $4: 36 \pm 0: 44$ & $4: 12$ & $4: 27 \pm 0: 26$ & $4: 24 \pm 0: 51$ & $4: 03 \pm 0: 33$ & $4: 39 \pm 0: 24$ & $4: 25 \pm 0: 32$ & $4: 18 \pm 0: 29$ \\
\hline $\begin{array}{c}5: 18 \pm 0: 11 \\
6: 49\end{array}$ & $6: 15$ & & & $6: 23 \pm 0: 46$ & $5: 12 \pm 1: 10$ & $5: 13 \pm 1: 10$ & $5: 33 \pm 0: 51$ \\
\hline $5: 29 \pm 0: 21$ & $5: 21 \pm 0: 23$ & $5: 20 \pm 0: 17$ & $5: 15 \pm 0: 17$ & $5: 14 \pm 0: 23$ & $5: 22 \pm 0: 16$ & $5: 15 \pm 0: 17$ & $5: 18 \pm 0: 22$ \\
\hline $5: 27 \pm 0: 24$ & $5: 31 \pm 0: 28$ & $5: 29 \pm 0: 19$ & $5: 20 \pm 0: 22$ & $5: 26 \pm 0: 22$ & $5: 23 \pm 0: 21$ & $5: 23 \pm 0: 17$ & $5: 21 \pm 0: 25$ \\
\hline $5: 35 \pm 0: 29$ & $5: 28 \pm 0: 21$ & $5: 26 \pm 0: 23$ & $5: 18 \pm 0: 20$ & $5: 30 \pm 0: 32$ & $5: 29 \pm 0: 25$ & $5: 26 \pm 0: 20$ & $5: 28 \pm 0: 19$ \\
\hline $5: 51 \pm 0: 31$ & $5: 43 \pm 0: 34$ & $5: 40 \pm 0: 25$ & $5: 30 \pm 0: 22$ & $5: 27 \pm 0: 22$ & $5: 34 \pm 0: 22$ & $5: 30 \pm 0: 20$ & $5: 34 \pm 0: 21$ \\
\hline $6: 02 \pm 0: 25$ & $6: 03 \pm 0: 27$ & $5: 40 \pm 0: 24$ & $5: 34 \pm 0: 23$ & $5: 42 \pm 0: 34$ & $5: 42 \pm 0: 26$ & $5: 39 \pm 0: 25$ & $5: 37 \pm 0: 27$ \\
\hline $6: 21 \pm 0: 40$ & $6: 20 \pm 0: 38$ & $6: 07 \pm 0: 31$ & $5: 53 \pm 0: 20$ & $6: 02 \pm 0: 39$ & $5: 49 \pm 0: 29$ & $5: 52 \pm 0: 21$ & $5: 54 \pm 0: 27$ \\
\hline $6: 38 \pm 0: 35$ & $6: 43 \pm 0: 42$ & $6: 36 \pm 0: 26$ & $6: 18 \pm 0: 27$ & $6: 39 \pm 0: 40$ & $6: 19 \pm 0: 28$ & $6: 18 \pm 0: 25$ & $6: 12 \pm 0: 33$ \\
\hline $7: 15 \pm 0: 37$ & $7: 07 \pm 0: 51$ & $6: 49 \pm 0: 39$ & $6: 46 \pm 0: 36$ & $6: 45 \pm 0: 39$ & $6: 45 \pm 0: 29$ & $6: 42 \pm 0: 38$ & $6: 36 \pm 0: 34$ \\
\hline $7: 28 \pm 0: 37$ & $7: 31 \pm 0: 41$ & $7: 31 \pm 0: 40$ & $7: 18 \pm 0: 40$ & $7: 34 \pm 0: 45$ & $7: 02 \pm 0: 28$ & $7: 17 \pm 0: 41$ & $7: 09 \pm 0: 51$ \\
\hline $8: 24 \pm 0: 38$ & $7: 59 \pm 0: 47$ & $8: 13 \pm 0: 50$ & $7: 34 \pm 0: 30$ & $8: 01 \pm 0: 57$ & $8: 12 \pm 0: 50$ & $7: 48 \pm 0: 44$ & $7: 49 \pm 0: 48$ \\
\hline $9: 19 \pm 1: 36$ & $9: 02 \pm 1: 29$ & $8: 29 \pm 1: 14$ & $8: 51 \pm 0: 59$ & $8: 42 \pm 1: 09$ & $7: 43 \pm 0: 35$ & $8: 37 \pm 0: 55$ & $9: 31 \pm 1: 33$ \\
\hline $9: 54 \pm 1: 01$ & & $10: 34 \pm 0: 49$ & $9: 26 \pm 1: 52$ & $10: 08$ & $9: 51 \pm 0: 44$ & $10: 39 \pm 1: 33$ & $9: 23 \pm 2: 01$ \\
\hline $11: 52$ & $13: 49$ & $13: 15$ & & & $8: 34$ & & $11: 47 \pm 2: 50$ \\
\hline $13: 52$ & & & & & & & \\
\hline
\end{tabular}


TABLE IV.—Results of the mixed-effects regression analyses for performance in age groups.

\begin{tabular}{|c|c|c|c|c|c|c|}
\hline & & Estimate & Standard error & df & $\mathrm{t}$ & $P$ value \\
\hline \multirow[t]{4}{*}{$25-29$ years } & Constant term $[200 \mathrm{~m}]$ & 151.62 & 0.83 & 1406.77 & 182.27 & $<0.0001$ \\
\hline & {$[\mathrm{sex}=$ women $]$} & 20.49 & 1.13 & 1396.91 & 17.98 & $<0.0001$ \\
\hline & {$[$ distance $=400 \mathrm{~m}]$} & 172.22 & 1.01 & 655.37 & 169.42 & $<0.0001$ \\
\hline & {$[\mathrm{sex}=$ women $] \times[$ distance $=400 \mathrm{~m}]$} & 13.17 & 1.46 & 690.41 & 8.98 & $<0.0001$ \\
\hline \multirow[t]{4}{*}{$30-34$ years } & Constant term $[200 \mathrm{~m}]$ & 154.74 & 0.89 & 1543.88 & 171.99 & $<0.0001$ \\
\hline & {$[\mathrm{sex}=$ women $]$} & 21.07 & 1.33 & 1552.44 & 15.76 & $<0.0001$ \\
\hline & {$[$ distance $=400 \mathrm{~m}]$} & 175.17 & 0.98 & 685.70 & 177.69 & $<0.0001$ \\
\hline & {$[$ sex $=$ women $] \times[$ distance $=400 \mathrm{~m}]$} & 15.40 & 1.51 & 726.49 & 10.15 & $<0.0001$ \\
\hline \multirow[t]{4}{*}{$35-39$ years } & Constant term $[200 \mathrm{~m}]$ & 159.68 & 1.06 & 1512.63 & 150.02 & $<0.0001$ \\
\hline & {$[\mathrm{sex}=$ women $]$} & 22.91 & 1.55 & 1510.47 & 14.69 & $<0.0001$ \\
\hline & {$[$ distance $=400 \mathrm{~m}]$} & 179.83 & 1.18 & 635.15 & 151.17 & $<0.0001$ \\
\hline & {$[\mathrm{sex}=$ women $] \times[$ distance $=400 \mathrm{~m}]$} & 16.33 & 1.81 & 670.09 & 8.98 & $<0.0001$ \\
\hline \multirow[t]{4}{*}{$40-44$ years } & Constant term $[200 \mathrm{~m}]$ & 163.22 & 1.15 & 1544.27 & 141.71 & $<0.0001$ \\
\hline & {$[$ sex $=$ women $]$} & 27.50 & & 1554.88 & 15.99 & $<0.0001$ \\
\hline & {$[$ distance $=400 \mathrm{~m}]$} & 184.21 & 1.27 & 760.23 & 144.07 & $<0.0001$ \\
\hline & {$[\mathrm{sex}=$ women $] \times[$ distance $=400 \mathrm{~m}]$} & 23.68 & 1.94 & 783.89 & 12.18 & $<0.0001$ \\
\hline \multirow[t]{4}{*}{$45-49$ years } & Constant term $[200 \mathrm{~m}]$ & 169.46 & 1.26 & 1438.36 & 133.96 & $<0.0001$ \\
\hline & {$[\mathrm{sex}=$ women $]$} & 27.56 & 1.93 & 1472.35 & 14.25 & $<0.0001$ \\
\hline & {$[$ distance $=400 \mathrm{~m}]$} & 191.76 & 1.41 & 717.71 & 135.86 & $<0.0001$ \\
\hline & {$[\mathrm{sex}=$ women $] \times[$ distance $=400 \mathrm{~m}]$} & 23.28 & 2.19 & 774.60 & 10.60 & $<0.0001$ \\
\hline \multirow[t]{4}{*}{$50-54$ years } & Constant term $[200 \mathrm{~m}]$ & 177.49 & 1.89 & 1255.19 & 93.64 & $<0.0001$ \\
\hline & {$[\mathrm{sex}=$ women $]$} & 32.62 & 2.72 & 1282.10 & 11.96 & $<0.0001$ \\
\hline & {$[$ distance $=400 \mathrm{~m}]$} & 202.95 & 2.20 & & 91.90 & $<0.0001$ \\
\hline & {$[\mathrm{sex}=$ women $] \times[$ distance $=400 \mathrm{~m}]$} & 27.82 & 3.12 & .35 & 8.90 & $<0.0001$ \\
\hline \multirow[t]{4}{*}{$55-59$ years } & Constant term $[200 \mathrm{~m}]$ & 189.38 & 1.98 & 994.49 & 95.35 & $<0.0001$ \\
\hline & {$[\mathrm{sex}=$ women $]$} & 37.33 & 2.87 & 1001.97 & 12.99 & $<0.0001$ \\
\hline & {$[$ distance $=400 \mathrm{~m}]$} & 214.33 & 1.86 & 540.98 & 115.21 & $<0.0001$ \\
\hline & {$[\mathrm{sex}=$ women $] \times[$ distan } & 29.38 & 2.67 & 545.29 & 10.97 & $<0.0001$ \\
\hline \multirow[t]{4}{*}{$60-64$ years } & Constant term [200 m] & 199.34 & 2.39 & 798.07 & 83.14 & $<0.0001$ \\
\hline & [sex=women] & 41.36 & & 791.78 & 11.43 & $<0.0001$ \\
\hline & {$[$ distance $=400 \mathrm{~m}]$} & 226.25 & 2.18 & 495.56 & 103.54 & $<0.0001$ \\
\hline & {$[\mathrm{sex}=$ women $] \times[$ distanc } & 31.80 & 3.17 & 484.01 & 10.03 & $<0.0001$ \\
\hline \multirow[t]{4}{*}{$65-69$ years } & Constant term $[200 \mathrm{~m}]$ & 215.32 & 2.94 & 661.64 & 73.09 & $<0.0001$ \\
\hline & {$[\mathrm{sex}=$ women $]$} & & 4.44 & 651.09 & 10.48 & $<0.0001$ \\
\hline & {$[$ distance $=400 \mathrm{~m}]$} & & 2.60 & 390.33 & 93.50 & $<0.0001$ \\
\hline & {$[\mathrm{sex}=$ women $] \times[$ distanc } & & 4.07 & 401.11 & 8.05 & $<0.0001$ \\
\hline \multirow[t]{4}{*}{ 70-74 years } & Constant term $[200 \mathrm{~m}]$ & 230.54 & 4.33 & 517.89 & 53.24 & $<0.0001$ \\
\hline & {$[\mathrm{sex}=$ women $]$} & 56.08 & 6.70 & 518.29 & 8.36 & $<0.0001$ \\
\hline & {$[$ distance $=400 \mathrm{~m}]$} & 263.66 & 4.15 & 343.46 & 63.48 & $<0.0001$ \\
\hline & {$[\mathrm{sex}=$ women $] \times[$ distanc } & 52.67 & 6.32 & 343.39 & 8.32 & $<0.0001$ \\
\hline \multirow{4}{*}{$75-79$ years } & Constant term $[200 \mathrm{~m}]$ & 257.43 & 8.00 & 281.57 & 32.17 & $<0.0001$ \\
\hline & {$[\mathrm{sex}=$ women $]$} & 61.64 & 12.36 & 285.59 & 4.98 & $<0.0001$ \\
\hline & {$[$ distance $=400 \mathrm{~m}]$} & 297.70 & 7.66 & 193.66 & 38.82 & $<0.0001$ \\
\hline & {$[\mathrm{sex}=$ women $] \times[$ distanc } & 77.40 & 13.32 & 215.75 & 5.80 & $<0.0001$ \\
\hline \multirow{4}{*}{$80-84$ years } & Constant term $[200 \mathrm{~m}]$ & 284.84 & 12.16 & 114.82 & 23.42 & $<0.0001$ \\
\hline & {$[\mathrm{sex}=$ women $]$} & 70.88 & 19.50 & 111.29 & 3.63 & $<0.0001$ \\
\hline & {$[$ distance $=400 \mathrm{~m}]$} & 333.10 & 12.35 & 71.55 & 26.95 & $<0.0001$ \\
\hline & {$[$ sex $=$ women $] \times[$ distance $=400 \mathrm{~m}]$} & 85.67 & 18.14 & 64.17 & 4.72 & $<0.0001$ \\
\hline \multirow[t]{4}{*}{$85-89$ years } & Constant term $[200 \mathrm{~m}]$ & 337.02 & 25.13 & 29.60 & 13.41 & $<0.0001$ \\
\hline & {$[\mathrm{sex}=$ women $]$} & 72.65 & 46.06 & 31.55 & 1.57 & 0.125 \\
\hline & {$[$ distance $=400 \mathrm{~m}]$} & 385.97 & 35.87 & 18.21 & 10.75 & $<0.0001$ \\
\hline & {$[$ sex $=$ women $] \times[$ distance $=400 \mathrm{~m}]$} & 290.69 & 65.41 & 20.42 & 4.44 & $<0.0001$ \\
\hline \multirow[t]{4}{*}{ 90-94 years } & Constant term $[200 \mathrm{~m}]$ & 418.77 & 28.72 & 7.33 & 14.57 & $<0.0001$ \\
\hline & {$[\mathrm{sex}=$ women $]$} & 17.52 & 35.17 & 7.33 & 0.49 & 0.633 \\
\hline & {$[$ distance $=400 \mathrm{~m}]$} & 420.07 & 32.78 & 3.79 & 12.81 & $<0.0001$ \\
\hline & {$[$ sex $=$ women $] \times[$ distance $=400 \mathrm{~m}]$} & 76.17 & 40.15 & 3.79 & 1.89 & 0.134 \\
\hline
\end{tabular}




\section{Performance trends}

Tables II and III summarize race times for women and men for $200 \mathrm{~m}$ and $400 \mathrm{~m}$ and Table IV the results of the mixed-effects regression analyses. For age groups 25-29 to $90-94$ years, women and men performance improved across years. For age groups 25-29 to $80-84$ years, men were faster than women. For age group 85-90 and 90-94 years, however, women were not slower than men. Sex and distance showed a significant interaction in all age groups from 25-29 to 85-89 years, but not for 90-94 years.

\section{Sex difference}

Figure 5 presents the trend in sex difference across years. In $200 \mathrm{~m}$, women reduced the sex difference. In $200 \mathrm{~m}$, there was a sex $\times$ calendar year interaction of trivial magnitude $\left(\mathrm{P}=0.025, \eta^{2}=0.003\right)$ on race time, where a larger improvement in women was noticed. A main effect of sex on race time $\left(\mathrm{P}<0.001, \eta^{2}=0.101\right)$ of medium magnitude was observed, where men were faster than women. Also, a main effect of calendar year on race time $(\mathrm{P}<0.001$, $\left.\eta^{2}=0.064\right)$ of medium magnitude was shown, where race time improved across years. In $400 \mathrm{~m}$, there was not any sex $\times$ calendar year interaction on race time $(\mathrm{P}=0.101$, $\left.\eta^{2}=0.004\right)$. A main effect of sex on race time $(\mathrm{P}<0.001$, $\left.\eta^{2}=0.101\right)$ of medium magnitude was observed, where men were faster than women $\left(\mathrm{P}<0.001, \eta^{2}=0.081\right)$. A main effect of calendar year on race time $\left(P<0.001, \eta^{2}=0.037\right)$ of small magnitude was shown, where race time improved across years. In summary, the sex and the calendar year had larger effect on race time in $200 \mathrm{~m}$ than in $400 \mathrm{~m}$.

\section{Discussion}

The aim of this study was to investigate trends in changes in participation, performance and sex differ-

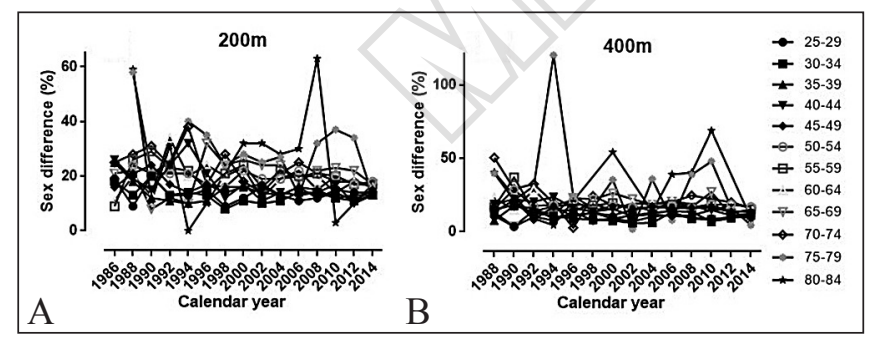

Figure 5.-A, B) Sex differences in performance by age group and calendar years. ence in performance in individual medley master swimmers competing in the FINA World Masters Championships in $200 \mathrm{~m}$ and $400 \mathrm{~m}$ with the hypothesis that participation would increase and performance would improve across years. The most important findings were: 1) the participation of swimmers older than 35 years in $200 \mathrm{~m}$ and of swimmers older than 50 years in $400 \mathrm{~m}$ increased; 2) more men competed in $200 \mathrm{~m}$ and $400 \mathrm{~m} ; 3$ ) women and men improved performance across years in all age groups from 25-29 to 90-94 years; 4) men were faster than women from 25-29 to 80-84 years, but not from 85-89 to 90-94 years; and 5) women reduced the gap to men only in age groups from 40-44 to 45-49 years.

Participation increased in women and men in the older age groups

A first finding was that participation in individual medley master swimmers increased in older age groups (>35 years in $200 \mathrm{~m}$ and $>50$ years in $400 \mathrm{~m}$ ). The youngest age group in the FINA World Masters Championships is $25-29$ years. ${ }^{1}$ It is very likely that swimmers in the younger age groups (i.e. younger than 30 years) preferably compete in races held at world class level such as the World Championships and the Olympic Games. 4, 19, 20 The fastest race times in elite swimmers are achieved at the age of $\sim 21-25$ years. ${ }^{19}$

The increase of participation in swimmers in older age groups might be explained by the increase in life expectancy in recent decades. ${ }^{21-24}$ Considering the World Population Ageing Report from 2013,25 the global share of older people (aged 60 years or over) increased from $9.2 \%$ in 1990 to $11.7 \%$ in 2013 and will continue to grow as a proportion of the world population, reaching $21.1 \%$ by 2050 . The older population is itself ageing. Globally, the share of older persons aged 80 years or over within the older population was $14 \%$ in 2013 and is projected to reach $19 \%$ in 2050 . The older population is predominantly women. Because women tend to live longer than men, older women outnumber older men almost everywhere. In 2013, globally, there were 85 men per 100 women in the age group 60 years or over and 61 men per 100 women in the age group 80 years or over. Due to improvements in health, 80 -year-old will have levels of both physical and mental capacities similar to that of many 20 -year-olds. ${ }^{26}$ 


\section{More men competed in $200 \mathrm{~m}$ and $400 \mathrm{~m}$}

The finding of more men than women competing in $200 \mathrm{~m}$ and $400 \mathrm{~m}$ was in agreement with previous swimming events. Forinstance, whenweconsider long-distance swimming events such as the English Channel Swim, ${ }^{27}$ the Catalina Channel Swim 28 or the Manhattan Island Marathon Swim, ${ }^{29}$ more men than women were competing. Also for shorter long-distance races such as $10 \mathrm{~km}$ swimming, men more than women were competing. ${ }^{30}$

\section{Women and men improved performance in all distances}

With regards to performance trends, women and men improved performance in all age groups and for all distances. This confirms previous findings for age group marathon runners 16 and age group swimmers. ${ }^{13}, 31$ However, this is the first study to show that octogenarians and nonagenarians improved performance in individual medley swimming. In the study of Akkari et al. ${ }^{13}$ investigating age groups swimmers and Lepers and Cattagni 16 investigating age group marathoners, the oldest swimmers considered were competing in age group 75-79 years. A very recent study investigating marathoners older than 75 years competing between 2004 and 2011 showed, however, that participation for women and men runners remained unchanged and the fastest women and men became slower across years. ${ }^{14}$

A potential explanation that these elderly swimmers improved performance could be their training. It has been shown that training distance in older master swimmer aged 52-82 years may be an important factor for maintaining muscle mass and function in the aging process. ${ }^{32}$ A study investigating French master swimmers showed positive health outcomes regarding different aspects such as weight management, respiratory function, and vitality due to their race preparation where positive health outcomes were higher for women master swimmers. ${ }^{33}$

\section{Women were not slower compared to men in age groups 85-89 to 90-94 years}

Considering the variation of sex differences by age group, men were faster than women in age groups 25 29 to 80-84 years, but not in age groups 85-89 to 90-94 years. The most likely explanation might be the very low number of women and men competitors older than 85 years. Another explanation might be anthropometric dif- ferences between elderly women and elderly men since there seemed to be differences between the sexes regarding an age-related loss in skeletal muscle mass. Men older than 70 years lose significantly more fat-free mass than women of the same age ${ }^{34}$ and in 68-78 years old women and men, the rate of loss in leg muscle was significantly higher in men than in women. ${ }^{35}$ With higher ages, sarcopenia occurs more frequently in men then in women. In women and men older than 80 years, the prevalence of sarcopenia was $-31 \%$ in women and $~ 53 \%$ in men. ${ }^{36}$

\section{Decrease of sex difference in middle age groups}

Another important finding was that women were able to reduce the gap to men only in the age groups $40-44$ to 45-49 years. This trend should not be attributed to any age-related physiological mechanism. The sex differences were decreased mostly in those age groups, which showed the largest differences in the first championships. Thus, the different trend of the age groups 40-44 and $45-49$ years from the others might be interpreted by their baseline values.

\section{Limitations and strengths of the study}

A limitation of this study was that the individual medley, despite being a combination of all swimming strokes, has certain physiological differences than the other strokes separately. ${ }^{37}$ Thus, caution is needed to generalize the finding of this study to the other strokes. On the other side, the present study is one of the few ever carried out on individual medley, and its findings might enhance the understanding of both sports scientists and swimming practitioners about major performance characteristics of this event.

\section{Conclusions}

In summary, in age group individual medley swimmers competing in age groups 25-29 to 90-94 years in the FINA World Masters Championships between 1986 and 2014, the participation increased in $200 \mathrm{~m}$ in swimmers older than 35 years, whereas in $400 \mathrm{~m}$ in swimmers older than 50 years; more men competed in $200 \mathrm{~m}$ and $400 \mathrm{~m}$; women and men improved performance in all in age groups from 25-29 to 90-94 years; men were faster than women from 25-29 to 80-84 years, but not from 8589 to 90-94 years; and women were only able in $40-44$ 
to 45-49 years to reduce the gap to men. These data suggest that especially elder (geriatric) master swimmers will continue to increase their participation and improve their performance in individual medley swimming.

\section{References}

1. FINA website [Internet]. Available from: www.fina.org [cited 2017, Nov 14]

2. Kucia-Czyszczoń K, Dybińska E, Ambrozy T, Chwała W. Factors determining swimming efficiency observed in among less skilled swimmers. Acta Bioeng Biomech 2013;15:115-24.

3. Wolfrum M, Rüst CA, Rosemann T, Lepers R, Knechtle B. The effect of course length on individual medley swimming performance in national and international Athletes. J Hum Kinet 2014;42:187-200.

4. König S, Valeri F, Wild S, Rosemann T, Rüst CA, Knechtle B. Change of the age and performance of swimmers across World Championships and Olympic Games finals from 1992 to 2013 - a cross-sectional data analysis. SpringerPlus 2014;3:652.

5. Nevill AM, Whyte GP, Holder RL, Peyrebrune M. Are there limits to swimming world records? Int J Sports Med 2007;28:1012-7.

6. Stanula A, Maszczyk A, Roczniok R, Pietraszewski P, Ostrowski A, Zajac A, et al. The development and prediction of athletic performance in freestyle swimming. J Hum Kinet 2012;32:97-107.

7. Buhl C, Knechtle B, Rust CA, Rosemann T, Lepers R. A comparison of medley and freestyle performance for national and international swimmers between 1994 and 2011. Open Access J Sports Med 2013;4:79-87.

8. Buhl C, Knechtle B, Rüst CA, Rosemann T, Lepers R. Women achieve peak swim performance in individual medley at earlier ages than men. Medicina Sportiva 2013;17:54-9.

9. Vaso M, Knechtle B, Rüst CA, Rosemann T, Lepers R. Age of peak swim speed and sex difference in performance in medley and freestyle swimming - A comparison between $200 \mathrm{~m}$ and $400 \mathrm{~m}$ in Swiss elite swimmers. J Hum Sport Exerc 2013;8:954-65.

10. Saavedra JM, Escalante Y, Garcia-Hermoso A, Arellano R, Navarro F. A 12 -year analysis of pacing strategies in 200- and 400-m individual medley in international swimming competitions. J Strength Cond Res 2012;26:3289-96.

11. Gatta G, Benelli P, Ditroilo M. The decline of swimming performance with advancing age: A cross-sectional study. J Strength Cond Res 2006;20:932-8.

12. Senefeld J, Joyner MJ, Stevens A, Hunter SK. Sex differences in elite swimming with advanced age are less than marathon running. Scand J Med Sci Sports 2016;26:17-28.

13. Akkari A, Machin D, Tanaka H. Greater progression of athletic performance in older Masters athletes. Age Ageing 2015;44:683-6.

14. Ahmadyar B, Rüst CA, Rosemann T, Knechtle B. Participation and performance trends in elderly marathoners in four of the world's largest marathons during 2004-2011. SpringerPlus. 2015;4:465.

15. Jokl P, Sethi PM, Cooper AJ. Master's performance in the New York City Marathon 1983-1999. Br J Sports Med 2004;38:408-12.

16. Lepers R, Cattagni T. Do older athletes reach limits in their performance during marathon running? Age 2012;34:773-81.

17. Zingg MA, Knechtle B, Rüst CA, Rosemann T, Lepers R. Analysis of participation and performance in athletes by age group in ultramarathons of more than $200 \mathrm{~km}$ in length. Int J Gen Med 2013;6:209-20.
18. Cohen J. Statistical power analysis for the behavioral sciences. Second edition. Hillsdale, NJ: Lawrence Erlbaum Associates; 1988.

19. Allen SV, Hopkins WG. Age of peak competitive performance of elite athletes: A systematic review. Sports Med 2015;45:1431-41.

20. Allen SV, Vandenbogaerde TJ, Hopkins WG. Career performance trajectories of Olympic swimmers: Benchmarks for talent development. Eur J Sport Sci 2014;14:643-51.

21. Manton KG, Vaupel JW. Survival after the age of 80 in the United States, Sweden, France, England, and Japan. New Engl J Med 1995:333:1232-5.

22. Murray CJL, Lopez AD. Alternative projections of mortality and disability by cause 1990-2020: Global Burden of Disease Study. Lancet 1997:349:1498-504.

23. Vernay M, Bonaldi C, Grémy I. Chronic diseases: Recent trends, challenges and prospects for change. Sante Publique 2015;27:S18997.

24. Vaupel JW, Carey JR, Christensen K, Johnson TE, Yashin AI, Holm NV, et al. Biodemographic trajectories of longevity. Sci 1998;280:855-60.

25. United Nations. World Population Aging 2013 [Internet]. Available from: www.un.org/en/development/desa/population/publications/ pdf/ageing/WorldPopulationAgeing2013.pdf [cited 2017, Nov 14]

26. World Health Organization. World Report on Aging and Health; 2015 [Internet]. Available from: www.who.int/kobe centre/mediacentre/ world report on ageing and health eng.pdf [cited 2017, Nov 14].

27. Eichenberger E, K̄nechtle B, Knechtle P, RüSt CA, Rosemann T, Lepers R. Best performances by men and women open-water swimmers during the 'English Channel Swim' from 1900 to 2010. J Sports Sci 2012;30:1295-301.

28. Knechtle B, Rosemann T, Rüst CA. Women cross the 'Catalina Channel' faster than men. SpringerPlus 2015;4:322.

29. Knechtle B, Rosemann T, Lepers R, Rüst CA. Women outperform men in ultradistance swimming: The Manhattan Island Marathon Swim from 1983 to 2013. Int J Sports Physiol Perform 2014;9:91324.

30. Vogt P, Rüst CA, Rosemann T, Lepers R, Knechtle B. Analysis of $10 \mathrm{~km}$ swimming performance of elite male and female open-water swimmers. SpringerPlus 2013;2:603.

31. Medic N, Young BW, Starkes JL, Weir PL, Grove JR. Gender, age, and sport differences in relative age effects among us masters swimming and track and field athletes. J Sports Sci 2009;27:1535-44.

32. Abe T, Kojima K, Stager JM. Skeletal muscle mass and muscular function in master swimmers is related to training distance. Rejuvenation Res 2014;17:415-21.

33. Potdevin F, Vanlerberghe G, Zunquin G, Peze T, Theunynck D. Evaluation of global health in master swimmers involved in French National Championships. Sports Med Open 2015;1:12.

34. Fantin F, Di Francesco V, Fontana G, Zivelonghi A, Bissoli L, Zoico E, Rossi A, Micciolo R, Bosello O, Zamboni M. Longitudinal body composition changes in old men and women: Interrelationships with worsening disability. J Gerontol A Biol Sci Med Sci 2007;62:137581 .

35. Zamboni M, Zoico E, Scartezzini T, Mazzali G, Tosoni P, Zivelonghi A, Gallagher D, De Pergola G, Di Francesco V, Bosello O. Body composition changes in stable-weight elderly subjects: The effect of sex. Aging Clin Exp Res 2003;15:321-7.

36. Iannuzzi-Sucich M, Prestwood KM, Kenny AM. Prevalence of sarcopenia and predictors of skeletal muscle mass in healthy, older men and women. J Gerontol A Biol Sci Med Sci 2002;57:M772-7.

37. Holmér I. Swimming physiology. Ann Physiol Anthropol 1992;11:269-76.

Conflicts of interest.- The authors certify that there is no conflict of interest with any financial organization regarding the material discussed in the manuscript. Article first published online: April 28, 2017. - Manuscript accepted: April 13, 2017. - Manuscript revised: March 23, 2017. - Manuscript received: September 8, 2016. 